Tropical Journal of Pharmaceutical Research May 2019; 18 (5): 1041-1047

ISSN: $1596-5996$ (print); 1596-9827 (electronic)

(C) Pharmacotherapy Group, Faculty of Pharmacy, University of Benin, Benin City, 300001 Nigeria.

\title{
Neuroprotective effect of thiamine triethylorthoformate conjugate against Parkinson disease in a mouse model
}

\author{
Yan Dong, Qing Xu, Xi Jia, Chao Li, Dan Xu, Yang Jiao* \\ Department of Neurology, Hong Qi Hospital Affiliated to MuDan Jiang Medical University, MuDan Jiang City, Heil Long Jiang \\ Province-157000, China
}

*For correspondence: Email: SacksMerlehem@yahoo.com; Tel/Fax: +86 4536582800

\begin{abstract}
Purpose: To investigate the effect of thiamine triethylorthoformate conjugate (TTO) on Parkinson disease $(P D)$ in vitro and in vivo in a mice model.

Methods: The effect of TTO on behavioural changes in PD mouse model was studied using pole, traction and swimming tests. Astrocyte proliferation after TTO treatment was assessed using 3 (4, 5 dimethyl 2 thi-azolyl) 2, 5 diphenyl $2 \mathrm{H}$ tetrazolium bromide (MTT) assay. Apoptosis was determined with flow cytometry using Annexin V Fluorescein isothiocyanate kit.

Results: Treatment of PD mice with TTO led to a decrease in climbing time, increase in suspension score and enhancement of swimming score, when compared to the untreated group $(p<0.05)$. Treatment of astrocytes with TTO prior to MPP incubation significantly increased proliferation $(p<0.05)$. Apoptosis induction in astrocytes by MPP was attenuated by pre-treatment with TTO. Pre-treatment of astrocytes with $10 \mu \mathrm{M}$ TTO markedly reduced JNK activation, when compared to astrocytes incubated with MPP alone $(p<0.05)$. Up-regulation of Bax and down-regulation of Bcl 2 by MPP in astrocytes were attenuated by pre-treatment with TTO. MPP-induced up-regulation of cleaved caspase 3 was suppressed in astrocytes by TTO pre-treatment $(p<0.05)$.

Conclusion: Treatment with TTO prevents MPP'-induced neuronal damage in vitro in astrocytes and in vivo in mice. The neuro-protective effect of TTO involves down-regulation of JNK activation, inhibition of caspase-3 level, decrease in Bax and increase in Bcl-2 expression. Thus, TTO has a potential for use in the treatment of Parkinson's disease.
\end{abstract}

Keywords: Thiamine-triethylorthoformate, Parkinson's disease, Neurodegeneration, Inflammation, Inflammation, Oxidative damage

\footnotetext{
This is an Open Access article that uses a fund-ing model which does not charge readers or their institutions for access and distributed under the terms of the Creative Commons Attribution License (http://creativecommons.org/licenses/by/4.0) and the Budapest Open Access Initiative (http://www.budapestopenaccessinitiative.org/read), which permit unrestricted use, distribution, and reproduction in any medium, provided the original work is properly credited.

Tropical Journal of Pharmaceutical Research is indexed by Science Citation Index (SciSearch), Scopus, International Pharmaceutical Abstract, Chemical Abstracts, Embase, Index Copernicus, EBSCO, African Index Medicus, JournalSeek, Journal Citation Reports/Science Edition, Directory of Open Access Journals (DOAJ), African Journal Online, Bioline International, Open-J-Gate and Pharmacy Abstracts
}

\section{INTRODUCTION}

Parkinson disease (PD) is the second most commonly detected neurodegenerative disorder with morbidity of $2 \%$ in aged people. It causes induction of apoptosis in basal ganglia leading to mental disturbance and bradykinesia in the patients [1]. Although the mechanism underlying PD is yet to be clearly understood, the disorder is associated with dysfunction of mitochondrion, neuronal inflammation and oxidative damage [2]. Increased levels of reactive oxygen species, glutamate and other cytotoxic molecules in the nervous system lead to neuronal inflammation 
and neurological degeneration through the onset of apoptosis [3].

Astrocyte dysfunction in mice critically affects the functioning and survival of neurons [4]. In animal models of brain injury, death of astrocytes generally proceeds through necrosis [5]. Astrocyte apoptosis plays an important role in the pathogenesis of neurodegenerative disorders such as Alzheimer's and Parkinson diseases [5]. Initially, 1-methyl-4-phenyl-1, 2, 3, 6-tetrahydropyridine (MPTP) was synthesized and used as an analgesic [6]. However, its administration to humans and rats has been found to cause Parkinson disease (PD) [7]. Administration of MPTP to mice causes neuronal death, and leads to symptoms of PD [8]. It is known that MPTP is a prodrug that gives rise to MPP+, a neurotoxin [9].

It is known that MPP+ causes degeneration of nigral dopaminergic neurons, and it can be used for the establishment of in vitro PD model [9]. In vitro and in vivo PD models have been established using MPP+ [10]. All symptoms of PD are produced by the administration of MPP+ in the cells as well as in animal models [10]. In the present study, the neuroprotective effect of thiamine-triethylorthoformate (TTO) conjugate was investigated in vitro on PD astrocytes, and in vivo in $\mathrm{PD}$ mice model.

\section{EXPERIMENTAL}

\section{Animals}

A total of 30 male BALB/c mice $(8-10$ weeks old, and weighing about $20 \mathrm{~g}$ each) were purchased from the Laboratory of Shanghai Animal Co. (SLAC, Shanghai, China). The mice were acclimatized to the laboratory environment at a temperature of $25 \pm 2{ }^{\circ} \mathrm{C}$ and humidity of 55 $\%$ under $12 \mathrm{~h}$ light/12h dark cycle. They were provided free access to standard laboratory diet and water. The animal experiments were carried out in accordance with the guidelines issued by the National Institutes of Health [11], with approval from the Committee for Animal Care and Use, Tongji University, Shanghai (approval no. STU/0017/103).

\section{Experimental design}

The mice were assigned randomly to six groups (5 mice per group): negative control, untreated (MPTP) and three treatment groups $(0.5 \mathrm{mg} / \mathrm{kg}$ TTO and MPTP; $1.0 \mathrm{mg} / \mathrm{kg}$ TTO and MPTP; 1.5 $\mathrm{mg} / \mathrm{kg}$ TTO and MPTP, and $2.0 \mathrm{mg} / \mathrm{kg}$ TTO and MPTP). The mice model of PD was prepared by injecting MPTP at a dose of $30 \mathrm{mg} / \mathrm{kg}$ daily for 5 days through the intra-peritoneal route. Thiamine-triethylorthoformate conjugate was injected to the mice in the treatment groups only on the first day, $45 \mathrm{~min}$ before MPTP administration. The mice in the treatment groups were injected with normal saline alone. Behavioural changes in the mice were assessed two days before, and 2, 4, 8 and 12 days after MPTP injection.

\section{Climbing test}

The pole test was used for the determination of climbing time of mice [11]. In this test, a ball was fixed over the top of a wooden pole about $50 \mathrm{~cm}$ in length and $1 \mathrm{~cm}$ width. The radius of the ball was $1.25 \mathrm{~cm}$, and on this ball the mice were placed so as to measure to time taken in getting down from it. If a mouse stopped or climbed in reverse direction, the experiment was repeated. The experiment was carried out three times every day for each mouse.

\section{Suspension test}

Traction test was used for determination of hang time of the mice [12]. In this test, the mice were suspended horizontally $30 \mathrm{~cm}$ above the ground using the hanging platform. The suspension score was determined using the following criteria: $0-4 \mathrm{~s}=0$ point; $5-9 \mathrm{~s}=1$ point; $10-14 \mathrm{~s}=2$ points; $15-19 \mathrm{~s}=3$ points; $20-24 \mathrm{~s}=4$ points; $25-29 s=5$ points, and above $30 s=6$ points.

\section{Determination of swimming time}

The mice were placed in a small swimming pool of dimensions $20 \times 30 \times 20 \mathrm{~cm}$. The temperature of the pool was adjusted to $30 \pm 2{ }^{\circ} \mathrm{C}$, and swim time was determined according to the procedure reported earlier [13]. The swimming score of mice was recorded over $1 \mathrm{~min}$ as follows: occasional swimming while floating mostly was scored 1 point; occasional swimming was scored 1.5 points, floating for more than half of the time was scored 2 points, swimming most of the time was scored 2.5 points, and successive swimming was scored 3 points.

\section{Astrocyte collection and culture}

The cerebral cortex of mice on postnatal day 5 was minced in DMEM medium supplemented with $20 \mu \mathrm{g} / \mathrm{mL}$ DNase and bovine serum albumin. Digestion of the tissues was achieved by treatment with $0.25 \%$ trypsin for 45 min at 37 ${ }^{\circ} \mathrm{C}$. Filtration of the tissue suspension was followed by centrifugation. Subsequently, the pellets were put into a DMEM medium containing 
FBS and $1 \%$ each of penicillin and streptomycin. The suspension was then transferred into the flasks and subjected to incubation under humidified atmosphere at $37{ }^{\circ} \mathrm{C}$. After reaching confluence, the microglia cells were removed from the flasks. The astrocytes were washed three times with PBS, trypsinized and then collected. Then, the astrocytes were cultured in DMEM/F12 separately at $37{ }^{\circ} \mathrm{C}$ under $5 \% \mathrm{CO}_{2}$ atmosphere. The PD astrocyte model was prepared by incubation in DMEM medium containing $4 \mathrm{mM} / \mathrm{L} \mathrm{MPP}+$. The astrocytes were cultured for $48 \mathrm{~h}$ in DMEM medium containing 1 , 2, 3, 4, 5, 6 and $10 \mu \mathrm{M}$ TTO.

\section{MTT assay}

The astrocytes at a density of $1 \times 10^{5}$ cells $/ \mathrm{ml}$ in $200 \mu \mathrm{L}$ of cell suspension were seeded in 96-well plates. After $24 \mathrm{~h}$ of incubation in DMEM medium, the cells were transferred to the new medium containing MPTP. The medium was mixed with TTO at concentrations of 1, 2, 3, 4, 5, 6 and $10 \mu \mathrm{M}$, and incubated for $48 \mathrm{~h}$. Then, MTT solution $(5 \mathrm{mg} / \mathrm{mL}, 20 \mu \mathrm{l})$ was added to each well of the plate and incubation was continued for $4 \mathrm{~h}$. The plates were washed in PBS, and treated with DMSO $(150 \mu \mathrm{l} /$ well). Absorbance was recorded for each well of the microtiter plate using Bio-Rad iMark plate reader at a wavelength of $455 \mathrm{~nm}$.

\section{Flow cytometric analysis}

Assessment of apoptosis induction in astrocytes was performed using Annexin V/FITC kit (BD Biosciences, San Jose, CA, USA) in accordance with the manufacturer's instructions. The astrocytes were collected after treatment and treated with HEPES binding buffer at room temperature for $25 \mathrm{~min}$. The buffer contained Annexin/V-FITC and PI. FACSCanto ${ }^{\mathrm{TM}}$ flow cytometer (BD Biosciences) was used for the analysis of cells and determination of apoptosis.

\section{Western blotting}

The astrocytes were lysed after washing with PBS, treated with pre-cold PBS, and subjected to homogenisation with RIPA lysis buffer containing inhibitors of protease and phosphatase. The lysate samples were collected and separated using gel electrophoresis on sodium dodecyl sulfate polyacrylamide (SDS-PAGE), and transferred onto polyvinylidene fluoride (PVDF) membrane (Millipore, Bedford, USA). The membrane non-specific sites were blocked by incubation with Tris-Buffered Saline and Tween20 (TBST). The membrane was then incubated over night at $4^{\circ} \mathrm{C}$ with primary antibodies against JNK, caspase-3, Bax and Bcl-2, with $\beta$-actin as control. The membrane was then washed twice with PBS and incubated for $1 \mathrm{~h}$ at room temperature with horse radish peroxidase (HRP)-conjugated secondary antibody.

\section{Statistical analysis}

Data are presented as mean \pm standard deviation of three experiments performed independently. Student's $t$-test and one-way ANOVA were used for determination of differences between the groups. The SPSS v17 software (SPSS, Inc., Chicago, IL, USA) was used for statistical analysis of the data. Differences were considered statistically significant at $p<0.05$.

\section{RESULTS}

\section{Movement defect in PD mice model was attenuated by TTO treatment}

Pole test showed that the time taken for climbing by the mice in negative control and PD groups was almost equal before and after 2 days of MPTP-administration (Figure 1). However, the time taken for climbing by the mice in untreated PD group increased markedly from day 6 to day 12 , when compared to the negative control mice. Pre-treatment of PD mice with TTO led to decrease in climbing time in dose-dependent manner, relative to untreated mice. The time taken for climbing by the mice in $2.0 \mathrm{mg} / \mathrm{kg}$ group on day 12 was almost equal to that of that of the negative control group.

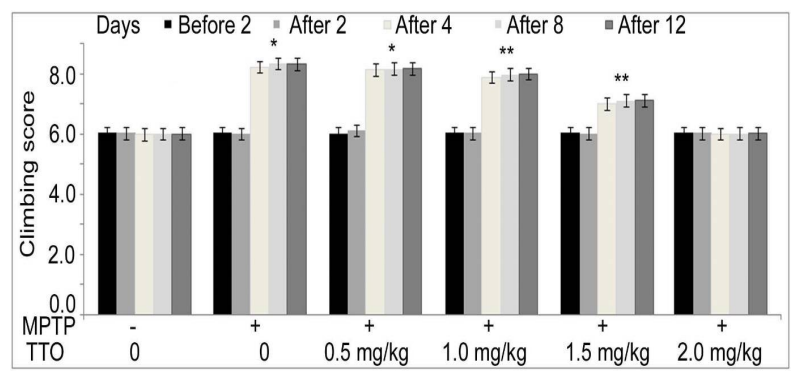

Figure 1: TTO treatment prevented MPTP-induced movement defect. The mice were injected 0.5, 1.0, 1.5 and $2.0 \mathrm{mg} / \mathrm{kg}$ doses of TTO or normal saline (untreated) prior to MPTP administration

\section{Effect of TTO treatment on suspension deficiency}

Traction test revealed that suspension score was almost equal for mice in the negative control and PD groups before and after 2 days of MPTPadministration (Figure 2). The suspension score was markedly lower for untreated PD mice from day 6 to day 12, when compared to the negative 
control mice. On the other hand, TTO treatment of PD mice increased the suspension score significantly, when compared to the untreated PD group. The suspension scores for mice in 2.0 $\mathrm{mg} / \mathrm{kg}$ TTO treatment group and that of mice in the negative control mice were almost similar on day 12 .

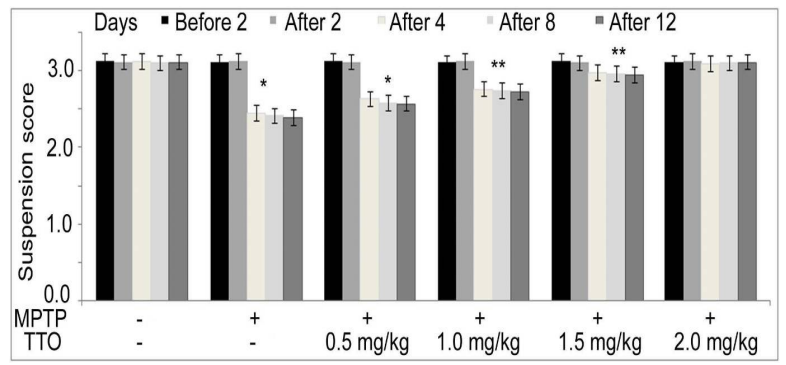

Figure 2: TTO-pre-treatment prevented MPTP-mediated decrease in suspension score. The mice were injected $0.5,1.0,1.5$ and $2.0 \mathrm{mg} / \mathrm{kg}$ doses of TTO or normal saline (untreated) prior to MPTP administration. Determination of suspension score was performed using traction test

\section{Effect of TTO treatment on swimming score}

Results from the swimming test revealed that swimming score was similar for the mice in the negative control and PD groups before and after 2 days of MPTP-administration (Figure 3 ). A significant decrease in the swimming score for untreated PD group was recorded from day 6 to day 12. In the PD mice treated with TTO, the swimming score was markedly increased, when compared to the untreated PD mice. Treatment of PD mice with $2.0 \mathrm{mg} / \mathrm{kg}$ TTO increased swimming score to the level of negative control group.

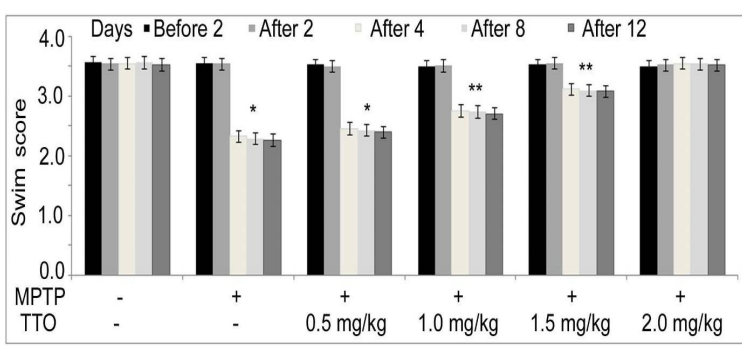

Figure 3: TTO-pre-treatment attenuated MPTP-mediated decrease in swimming score. The mice were injected with TTO at doses of $0.5,1.0,1.5$ and $2.0 \mathrm{mg} / \mathrm{kg}$ doses, or with normal saline (untreated) prior to MPTP administration. Swimming score was measured for all the mice

Treatment with TTO attenuated inhibition of primary astrocyte proliferation by MPTP

Treatment of astrocytes with TTO showed no effect on primary astrocyte proliferation, when compared with the negative control cells (Figure 4). However, the proliferation of astrocytes was significantly decreased on incubation with MPP. Pre-treatment of astrocytes with TTO attenuated the effect of MPP on proliferation. The proliferation of astrocytes incubated with MPP increased significantly on pre-treatment with TTO, when compared cultures incubated with MPP alone. Moreover, TTO attenuated the effect of MPP on proliferation in a concentration-based manner. The astrocytes were pre-treated with 1 , 2, 3, 4, 5, 6 and $10 \mu \mathrm{M}$ TTO for $48 \mathrm{~h}$ and then incubated with MPP. The effect of MPP on astrocyte proliferation was completely inhibited on treatment with $10 \mu \mathrm{M}$ TTO.

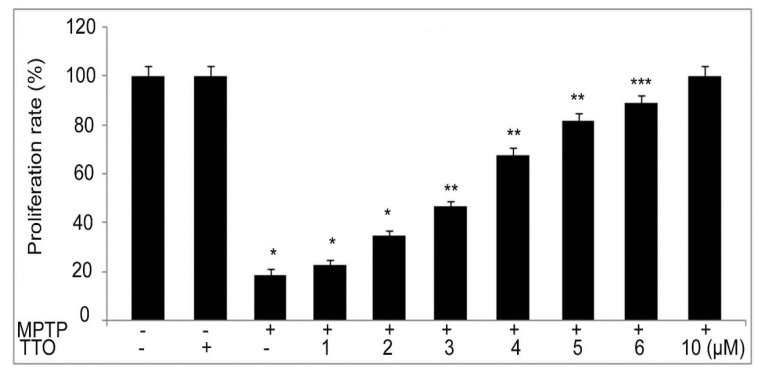

Figure 4: TTO inhibited MPP-mediated decrease in astrocyte proliferation. Astrocytes were treated with 1, $2,3,4,5,6$ and $10 \mu \mathrm{M}$ TTO for $48 \mathrm{~h}$ prior to incubation with MPTP. The negative control astrocytes were incubated with DMSO alone; ${ }^{*} p<0.05,{ }^{* *} p<0.02$ and ${ }^{* * *} p<0.01$, relative to negative control

\section{TTO inhibited apoptosis induction in astrocytes by MPP}

Incubation of astrocytes with MPP significantly induced apoptosis. However, TTO pre-treatment attenuated the apoptosis-inducing effect of MPP on astrocytes. The apoptosis inducing effect of MPP was completely attenuated by pretreatment of astrocytes with $10 \mu \mathrm{M}$ TTO for $48 \mathrm{~h}$ (Figure 5).
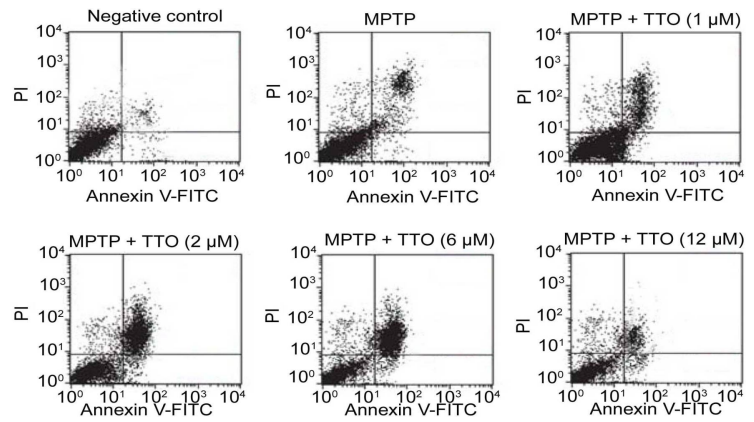

Figure 5: TTO blocked apoptosis induction by MPP in astrocytes. Astrocytes were treated with 1, 2, 3, 4, 5, 6 and $10 \mu \mathrm{M}$ TTO for $48 \mathrm{~h}$ prior to incubation with MPTP. The negative control astrocytes were incubated with DMSO alone. Annexin V-FITC/PI staining was used for the assessment of apoptosis 
TTO regulated activation of JNK and $\mathrm{Bax} / \mathrm{Bcl}-2$ ratio in astrocytes

Western blotting showed up-regulation of JNK in astrocytes on exposure to MPP. The JNK activation was decreased on pre-treatment with TTO for $48 \mathrm{~h}$. Pre-treatment of astrocytes with 10 $\mu \mathrm{M}$ TTO reduced JNK activation to the minimum level, when compared to astrocytes incubated with MPP alone (Figure 6). Incubation of astrocytes with MPP led to a marked increase in the expression of Bax, and significant decrease in $\mathrm{Bcl}-2$ level (Figure 6). However, in the TTO pre-treated astrocytes, the effect of MPP on Bax and $\mathrm{Bcl}-2$ was reversed: TTO pre-treatment of astrocytes for $48 \mathrm{~h}$ decreased Bax and increased $\mathrm{Bcl}-2$ expressions, when compared to those cultured with MPP alone. Thus, TTO pretreatment regulated the ratio of $\mathrm{Bax} / \mathrm{Bcl}-2$ in the astrocytes.

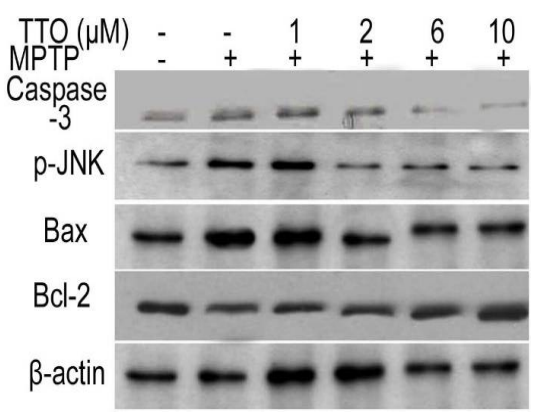

Figure 6: TTO treatment attenuated $\mathrm{Bax} / \mathrm{Bcl}-2$ ratio and JNK activation. Astrocytes were treated with 1, 2, 3, 4, 5, 6 and $10 \mu \mathrm{M}$ TTO for $48 \mathrm{~h}$ prior to incubation with MPTP. The negative control astrocytes were incubated with DMSO alone. Western blotting was used for the assessment of Bax, Bcl-2 and JNK expressions in astrocytes

\section{TTO regulated caspase-3 level in astrocytes}

The expression of cleaved caspase- 3 was increased markedly in astrocytes on incubation with MPP (Figure 7). However, TTO pretreatment of astrocytes decreased MPP-induced up-regulation of cleaved caspase-3. The decrease in the expression of cleaved caspase- 3 was maximum in the astrocyte cultures pretreated with $10 \mu \mathrm{M}$ TTO.

\section{DISCUSSION}

The present study investigated the effect of TTO on PD in a mouse model in vivo, and on astrocyte proliferation and apoptosis in vitro. PD mouse model was prepared by administration of MPP to mice using established methods [11-13]. It has been reported that mice with PD took more time in pole climbing test and scored low in swimming test, when compared to normal mice
[11-13]. It is has also been reported that in suspension test, the time taken by the PD mice was less than the time taken by normal mice [1113].

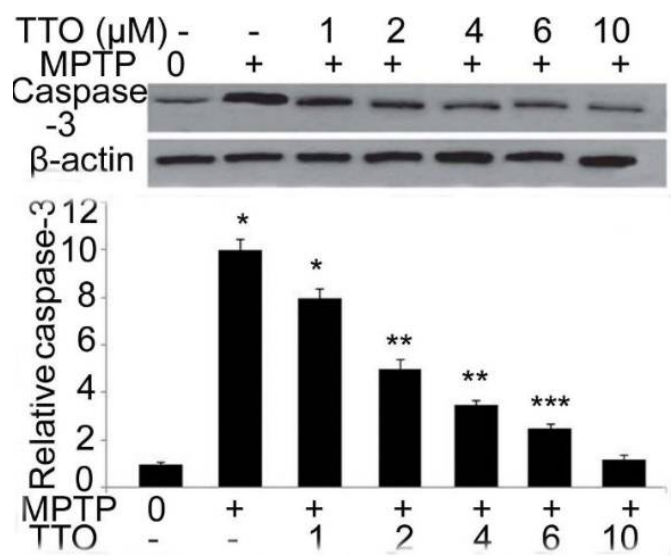

Figure 7: Regulation of caspase-3 level by TTO. After incubation with 1, 2, 3, 4, 5, 6 and $10 \mu \mathrm{M}$ TTO for $48 \mathrm{~h}$, astrocytes were cultured in a medium containing MPTP. The negative control astrocytes were incubated with DMSO alone. (A) Caspase-3 expression in astrocytes, as determined with Western blotting; (B) Quantification of the expression level of caspase-3; ${ }^{*} p<0.05,{ }^{* *} p<0.02$ and ${ }^{* * *} p<0.01$, relative to negative control

In the present study, MPP administration to mice led to increase in pole climbing time and increase in swimming score from day 2 , when compared to the negative control group. The suspension score was lower in mice administered MPP than in mice in the negative control group. These findings confirmed the establishment of PD mouse model after MPP administration. Treatment of the PD mice with TTO led to decrease in climbing time, relative to untreated mice.

At higher concentrations of TTO, the time taken for climbing by the mice was almost equal to that taken by mice in negative control group. The swimming score for PD mice increased significantly on treatment with TTO, when compared with that of mice in untreated group. In addition, TTO treatment of PD mice significantly increased the suspension score, when compared to the untreated PD group. The suspension scores for TTO-treated PD mice and the negative control group were almost equal on day 12 . Therefore, TTO treatment of PD mice led to a marked improvement in the MPP-induced behavioural changes.

In order to understand the mechanism involved in the improvement of MPP-induced behavioural changes in mice model by TTO, the astrocytes were treated with TTO for $48 \mathrm{~h}$ prior to incubation with MPP. Incubation of astrocytes with MPP 
caused a significant decrease in proliferation, when compared to the negative control cells. On the other hand, TTO pre-treatment of astrocytes prevented MPP-induced decrease in proliferation. Moreover, the apoptosis induction in astrocytes on incubation with MPP was also inhibited by TTO pre-treatment. Thus, TTO protected the astrocytes against MPP-induced cytotoxicity.

Activation of JNK pathway has been found to be associated with the stress induced apoptosis such as removal of nerve growth factor $[14,15]$. The activation of JNK pathway has been reported in mice after MPTP administration $[16,17]$. Phosphorylation of JNK leads to the induction of cell apoptosis [18]. In the present study, incubation of astrocytes with MPTP led to a marked increase in JNK activation. The expression of $\mathrm{p}$-JNK was found to be higher in the astrocytes incubated with MPTP. However, TTO treatment of astrocytes prior to incubation with MPTP decreased the phosphorylation of JNK. These results suggest that TTO inhibited apoptosis induction in astrocytes through downregulation of JNK-pathway.

Studies have shown that incubation of the astrocytes with MPTP led to activation of the tumor-suppressor protein, p53 which in turn interacts with JNK to promote the expression of Bax $[19,20]$. In the present study, incubation of astrocytes with MPP + increased the expression of Bax and decreased the level of Bcl-2. This led to increase in the ratio of $\mathrm{Bax} / \mathrm{Bcl}-2$ in astrocytes by incubation with MPP+. Increases in the ratio of $\mathrm{Bax} / \mathrm{Bcl}-2$ have been reported to induce apoptosis in astrocytes after incubation with MPP+ [21]. Pre-treatment of the astrocytes with TTO prior to incubation with MPP+ decreased Bax expression and enhanced Bcl-2 expression level. Thus, TTO pre-treatment regulated the $\mathrm{Bax} / \mathrm{Bcl}-2$ ratio in the astrocytes incubated with $\mathrm{MPP}+$. It appears that TTO regulated $\mathrm{Bax} / \mathrm{Bcl}-2$ ratio through the down-regulation of JNK phosphorylation.

During cell apoptosis, the level of activated caspase-3 is markedly higher than its level in normal cells [22]. In the present study, the level of activated caspase- 3 was found to be higher in astrocytes incubated with MPP+ than in negative control astrocytes. However, TTO treatment of the astrocytes prior to incubation with MPP+ inhibited activation of caspase-3.

\section{CONCLUSION}

These results demonstrate that TTO prevents MPP+-induced neuronal damage in vivo in a mice model, and in vitro in astrocytes. The neuro-protective effect of TTO involves downregulation of JNK activation, inhibition of caspase-3 activation, decrease in Bax level and increase in Bcl-2 expression. Thus, TTO may be suitable for the management of Parkinson disease.

\section{DECLARATIONS}

\section{Acknowledgement}

The authors are highly thankful to Head, Department of Neurology, Hong Qi Hospital affiliated to MuDan Jiang Medical University, MuDan Jiang City for his support.

\section{Conflict of interest}

No conflict of interest is associated with this work.

\section{Contribution of authors}

We declare that this work was done by the authors named in this article and all liabilities pertaining to claims relating to the content of this article will be borne by the authors. Yan Dong, Qing $\mathrm{Xu}$ and $\mathrm{Xi}$ Jia performed all the experimental work and made literature survey. Chao $\mathrm{Li}$ and Dan Xu analysed the data. All the authors compiled the data. Yang Jiao designed the study and wrote the manuscript. The research article was approved for communication by all the authors.

\section{Open Access}

This is an Open Access article that uses a funding model which does not charge readers or their institutions for access and distributed under the terms of the Creative Commons Attribution License (http://creativecommons.org/licenses/by/ 4.0) and the Budapest Open Access Initiative (http://www.budapestopenaccessinitiative.org/rea d), which permit unrestricted use, distribution, and reproduction in any medium, provided the original work is properly credited.

\section{REFERENCES}

1. Franco Iborra $S$, Vila $M$, Perier $C$. The Parkinson disease mitochondrial hypothesis: Where are we at? Neuroscientist 2016; 22: 266277.

2. Camilleri A, Vassallo $N$. The centrality of mitochondria in the pathogenesis and treatment of Parkinson's disease. CNS Neurosci Ther 2014; 20: 591602.

Trop J Pharm Res, May 2019; 18(5): 1046 
3. Takeuchi H, Mizuno T, Zhang G, Wang J, Kawanokuchi J, Kuno R, Suzumura A. Neuritic beading induced by activated microglia is an early feature of neuronal dysfunction toward neuronal death by inhibition of mitochondrial respiration and axonal transport. J Biol Chem 2005; 280: 1044410454.

4. Seifert G, Schilling $K$, Steinhauser C. Astrocyte dysfunction in neurological disorders: $A$ molecular perspective. Nat Rev Neurosci 2006; 7: 194206.

5. Kobayashi K, Hayashi M, Nakano H, Fukutani Y, Sasaki K, Shimazaki M, Koshino Y. Apoptosis of astrocytes with enhanced lysosomal activity and oligodendrocytes in white matter lesions in Alzheimer's disease. Neuropathol Appl Neurobiol 2002; 28: 238251.

6. Lee J, Ziering A, Heineman SD, Berger L. Piperidine derivatives; 2 phenyl and 2 phenylalkyl piperidines. $J$ Org Chem 1947; 12: 885893.

7. Pereira EAC, Aziz TZ. Parkinson's disease and primate research: past, present, and future. Postgraduate medical journal 2006; 82: 293-299.

8. Porras G, Li Q, Bezard E. Modeling Parkinson's disease in primates: the MPTP model. Cold Spring Harbor perspectives in medicine 2012; 2: a009308.

9. Seniuk NA, Tatton WG, Greenwood CE. Dose dependent destruction of the coeruleus cortical and nigral striatal projections by MPTP. Brain Res 1990; 527: 720.

10. Przedborski S, Jackson Lewis V. Mechanisms of MPTP toxicity. Mov Disord 1998; 13 (Suppl 1): S35 S38.

11. Ogawa $N$, Hirose $Y$, Ohara $S$, Ono $T$, Watanabe $Y$. A sireple quantitative hradykinesia test in MPIP treated mice. Res Commun Chem Pathol Pharmacol 1985; 50 435441.

12. Kubara $H$, Higuchi $Y$, Tadokoro S. Effects of central depressants on rota rod and action performances in mice. Jpn J Pharmacol 1977; 27: 117126.

13. Donnan GA, Willjs GL, Kaczmarczyk SJ, Rowe P. Motor function in the I, methyl 4 phenyl I,2,3,6 tetrahydropyridine treated mouse. J Neurol Sci 1987; 77: 185191.
14. Davis RJ. Signal transduction by the JNK group of MAP kinases. Cell 2000; 103: 239252.

15. Dickens M, Rogers JS, Cavanagh J, Raitano A, Xia Z, Halpern JR, Greenberg ME, Sawyers CL, Davis RJ. A cytoplasmic inhibitor of the JNK signal transduction pathway. Sci 1997; 277: 693696.

16. Saporito MS, Thomas BA, Scott RW. MPTP activates $C$ Jun $N H(2)$ terminal kinase (JNK) and its upstream regulatory kinase MKK4 in nigrostriatal neurons in vivo. J Neurochem 2000; 75: 12001208.

17. Xia XG, Harding $T$, Weller $M$, Bieneman $A$, Uney JB, Schulz JB. Gene transfer of the JNK interacting protein 1 protects dopaminergic neurons in the MPTP model of Parkinson's disease. Proc Natl Acad Sci USA 2001; 98 : 1043310438.

18. Lotharius J, Falsig J, van Beek J, Payne S, Dringen R, Brundin $P$, Leist $M$. Progressive degeneration of human mesencephalic neuron derived cells triggered by dopamine dependent oxidative stress is dependent on the mixed lineage kinase pathway. J Neurosci 2005; 25: 63296342

19. Findley HW, Gu L, Yeager AM, Zhou M. Expression and regulation of $\mathrm{BCl} 2, \mathrm{BCl} \times \mathrm{l}$, and Bax correlate with p53 status and sensitivity to apoptosis in childhood acute lymphoblastic leukemia. Blood 1997; 89: 29862993.

20. Mandir AS, Przedborski S, JacksonLewis V, Wang ZQ, Simbulan Rosenthal CM, Smulson ME, Hoffman BE, Guastella DB, Dawson VL, Dawson TM. Poly(ADP ribose) polymerase activation mediates 1 methyl 4 phenyl 1, 2,3,6 tetra-hydropyridine (MPTP) induced parkinsonism. Proc Natl Acad Sci USA 1999; 96: 5774 5779.

21. Blum D, Torch S, Lambeng N, Nissou M, Benabid AL, Sadoul R, Verna JM. Molecular pathways involved in the neurotoxicity of 6 OHDA, dopamine and MPTP: Contribution to the apoptotic theory in Parkinson's disease. Prog Neurobiol 2001; 65: 135172.

22. Salvesen GS. Caspases: Opening the boxes and interpreting the arrows. Cell Death Differ 2002; 9: 35. 